\title{
The Rates of Type I X\#Ray Bursts from Transients Observed withRXTE: Evidence for Black Hole Event Horizons
}

\section{Citation}

Remillard, Ronald A., Dacheng Lin, Randall L. Cooper, and Ramesh Narayan. 2006. “The Rates of Type I X\#Ray Bursts from Transients Observed withRXTE: Evidence for Black Hole Event Horizons." The Astrophysical Journal 646 (1): 407-19. https://doi.org/10.1086/504862.

\section{Permanent link}

http://nrs.harvard.edu/urn-3:HUL.InstRepos:41384866

\section{Terms of Use}

This article was downloaded from Harvard University's DASH repository, and is made available under the terms and conditions applicable to Other Posted Material, as set forth at http:// nrs.harvard.edu/urn-3:HUL.InstRepos:dash.current.terms-of-use\#LAA

\section{Share Your Story}

The Harvard community has made this article openly available. Please share how this access benefits you. Submit a story.

Accessibility 


\title{
The Rates of Type I X-ray Bursts from Transients Observed with RXTE: Evidence for Black Hole Event Horizons
}

\author{
Ronald A. Remillard and Dacheng Lin \\ MIT Kavli Institute for Astrophysics and Space Research, MIT, Cambridge, MA 02139-4307 \\ rr@space.mit.edu; lindc@space.mit.edu \\ and \\ Randall L. Cooper and Ramesh Narayan \\ Harvard-Smithsonian Center for Astrophysics, 60 Garden Street, Cambridge, MA 02138 \\ rcooper@cfa.harvard.edu, rnarayan@cfa.harvard.edu
}

\begin{abstract}
We measure the rates of type I X-ray bursts from a likely flux-limited sample of 37 non-pulsing Galactic transients observed with RXTE during 1996-2004. These sources are well-categorized in the literature as either neutron-star systems or black hole candidates. Our goals are to test the burst model for neutron stars and to investigate whether black holes have event horizons. Target selection is one of several differences between the present study and the investigation of the event horizon question by Tournear et al. (2003). Burst rates are measured as a function of bolometric luminosity, and the results are compared with augmented versions of the burst model developed by Narayan \& Heyl $(2002 ; 2003)$. For a given mass, we consider a range in both the radius and the temperature at the boundary below the accretion layer. We find 135 spectrally-confirmed type I bursts in 3.7 Ms of PCA exposures for the neutron-star group (13 sources), and the burst rate function is generally consistent with the model predictions. However, for the black hole groups (18 sources), there are no confirmed type I bursts in $6.5 \mathrm{Ms}$ of exposure, and the upper limits in the burst function are inconsistent with the model predictions for heavy compact objects with a solid surface. The consistency probability is $\sim 2 \times 10^{-7}$ for dynamical black-hole binaries, falling to $3 \times 10^{-13}$ with the added exposures of black-hole candidates. Furthermore, there are systematic spectral differences between the neutron-star and black-hole groups, supporting the presumption that physical differences underly the classifications in our sample. These results provide indirect evidence that black holes do have event horizons.
\end{abstract}

Subject headings: neutron star physics — black hole physics — general relativity — X-rays: stars

\section{Introduction}

Do black holes really have event horizons as described by general relativity? This question has been addressed using several different methods that judiciously compare the properties of black holes in accreting binary systems versus accreting neutron stars. Such investigations utilize the logic that the hard surface of a neutron star imposes a final boundary condition on accretion processes, and that this condition causes observational signatures that may be absent for black holes with event horizons (see review by Narayan 2003). The inherent difficulty in gaining inferences for a negative condition, i.e. that black holes have no surface, requires that this hypothesis be tested with an accretion process that can be tied to a detailed and reliable physical model. The confidence level for the inference of the event horizon can be increased by investigating this question with as many inde- 
pendent methods as possible.

Investigations of event horizons may capitalize on the fact that there are two classes of compact objects, distinguished by mass (Charles \& Coe 2006; McClintock \& Remillard 2006; Thorsett \& Chakrabarty 1999). Dynamical studies of the companion stars in some X-ray binary systems reveal compact objects with masses $\left(4-14 M_{\odot}\right)$ that are well above the theoretical upper limit for neutron stars (Kalogera \& Baym 1996). These are designated as "dynamical black hole" systems. We adopt this common convention and refer to these compact objects, henceforth, as black holes ("BH"), although for the purposes of this paper we consider them to be heavy compact objects that may have collapsed within an event horizon. The other group consists of neutron stars ("NS"). In practice, compact objects are routinely identified as NS systems if they display either coherent pulsations or type I X-ray bursts (see below). Dynamical measurements of NS binaries are led by the double-pulsar systems detected in the radio band, supplemented by a handful of X-ray pulsars and bursters (Charles \& Coe 2006; Thorsett \& Chakrabarty 1999). The results are all consistent with a mass near $1.4 M_{\odot}$, except for two X-ray pulsars which have an estimated mass near 2.0 $M_{\odot}$. Some non-pulsing Xray sources exhibit the brightness and variability characteristics of accreting compact objects, but their type is unknown, with no record of bursts nor measurements of binary motions, usually because of optically extincted or very faint companion stars. An important subgroup of such cases are known to exhibit the X-ray spectral states of black-hole binaries (BHBs), and they are labeled as black-hole candidates (BHCs), with a confidence level based on the extent of the observation archive and the clarity of $\mathrm{BH}$ states recorded (McClintock \& Remillard 2006).

A few different techniques have been used to gain evidence for $\mathrm{BH}$ event horizons. The first method focused on the quiescent state of X-ray transients, when there is a very low rate of mass transfer from the donor star to the accretion disk surrounding the compact object. Quiescent BHs were found to exhibit a systematically lower Xray luminosity, compared to quiescent NS transients (Narayan et al. 1997; Menou et al. 1999; Garcia et al. 2001; McClintock et al. 2004). An exception to this trend has been found in the observation of a quiescent NS system with very low luminosity (Jonker et al. 2006). The inefficient, non-thermal X-ray spectrum for quiescent BHs was interpreted by a model (Narayan \& Yi 1995) for an advection-dominated accretion flow (ADAF). This model explains the lack of a soft thermal component for quiescent BHs (McClintock et al. 2004), while the impact of the flow with a hard surface explains the higher luminosity and thermal X-ray spectrum observed for quiescent NS systems. This topic remains under investigation with evolution in the advection model (Narayan et al. 2000; Quataert \& Gruzinov 2000; Igumenshchev et al. 2003; Narayan \& Quataert 2005) and controversies about alternative accretion mechanisms such as a BH jet (Fender et al. 2004).

More recently, searches for evidence of event horizons have been initiated for the active states of accretion in X-ray binary systems. Narayan \& Heyl $(2002 ; 2003)$ showed that type I X-ray bursts can be used to probe the accumulation of accreted mass onto the surface of a compact object, and they found ways to predict how the burst rate (versus mass accretion rate) would vary as a function of the mass of the compact object. X-ray bursts are detected as bright and distinctive flares with 10-100 s duration in the X-ray band. They represent thermonuclear explosions on the surface of a NS (Strohmayer \& Bildsten 2006). For a wide range of parameters, type I bursts are an inevitable consequence of the accumulation of matter that is rich in $\mathrm{H}$ and $\mathrm{He}$ onto the ultra-dense and hot surface of a NS, or any hypothesized compact object with a solid surface and comparable scales of mass and radius.

A third variant of the search for $\mathrm{BH}$ event horizons focuses on the energy spectra of X-ray binaries in full outburst (Done \& Gierliński 2003). When the patterns of spectral evolution are examined, a distinct type of soft X-ray spectrum is occasionally observed, but only for BHBs and BHCs. This spectrum is understood as thermal emission from the inner accretion disk. It is argued that NSs with a high accretion rate cannot exhibit such a simple, low-temperature spectrum because of the energetics in a boundary layer that forms where accreting matter impacts the surface. The current level of confidence for the boundarylayer model may not be very high, given that so 
many details of accretion physics are complex and poorly understood. However, there do appear to be systematic differences in the X-ray spectra of accreting NSs vs. BHBs at high luminosity, and these differences are surely worth investigating in the context of the reality of $\mathrm{BH}$ event horizons.

In this paper, we offer new X-ray data analyses that investigate the destination of accreted matter by measuring the rates of type I X-ray bursts (hereafter "bursts") in X-ray binary systems. We compare the results to augmented versions of the burst model developed by Narayan \& Heyl (2002; 2003). A statistical study of burst rates in NS systems and BHBs was conducted by Tournear et al. (2003; "T03"). They argue that the mean burst rate for $\mathrm{BHBs}$ and $\mathrm{BHCs}$ is indeed far below the rate expected if $\mathrm{BHs}$ are heavy compact objects that do have a hard surface. In the present paper, we re-address this question and offer several differences with respect to the investigation of T03. 1.) Sources are selected from a flux-limited list of X-ray transients derived from 9 years of operation by the All-Sky Monitor (ASM) on the Rossi X-ray Timing Explorer ( RXTE ). The overlap with T03 for BHBs and BHCs is, in fact, rather small. 2.) We conduct computer searches for bursts, rather than "visual inspection", in order to carefully screen all burst candidates and to measure statistical distributions in parameters such as the detection threshold and the durations of all confirmed and rejected burst candidates. 3.) We measure the burst rates (and upper limits) as a function of the unabsorbed bolometric luminosity, scaled to the Eddington luminosity, in order to most effectively utilize burst models for both NSs and heavy compact objects.

Physical models for type I X-ray bursts have a rich and complex history (Woosley \& Taam 1976; Fujimoto, Hanawa, \& Miyaji 1981; Paczynski 1983; Fushiki \& Lamb 1987; Taam, Woosley, \& Lamb 1996; Cumming \& Bildsten 2000; Narayan \& Heyl 2002; 2003; Woosley et al. 2004). Current theory predicts three different burst regimes that are delimited by the mass accretion rate. Abundance changes due to the stable nuclear burning of $\mathrm{H}$ and/or $\mathrm{He}$ determine the properties of the bursts in each regime. There are some successes and many problems in applying burst models to explain the distributions in the burst timescale and amplitude in each regime (Kuulkers et al. 2002; Cornelisse et al. 2003; Strohmayer \& Bildsten 2006). Nevertheless, there are continuing improvements in the manner in which stable nuclear burning is handled (Narayan \& Heyl 2003; Woosley et al. 2004). This study requires a burst model that can reasonably predict the burst rate function for NS systems, and our sample group of NS transients provides critical feedback for this purpose.

The three goals of this study are: to determine whether the burst rates for non-pulsing NS systems match the predictions of the burst model for $\mathrm{NSs}$, to search for X-ray bursts in $\mathrm{BH}$ systems, and to determine whether the burst rates or upper limits for BHBs and BHCs are compatible with predictions of the burst model for heavy compact objects with a solid surface. In $\S 2$, we describe our selections of X-ray targets and RXTE pointed observations. The methods to identify burst candidates from X-ray timing analysis and to confirm bursts with X-ray spectral analysis are explained in $\S 3$. Details regarding the burst model are given in $\S 4$. The burst rates and comparisons with the model predictions are given in $\S 5$, followed by discussions and conclusions.

\section{Observations}

\subsection{ASM Sample of X-ray Transients, 1996-2004}

Comparative studies of the $\mathrm{BH}$ and NS classes of X-ray binary systems must contend with the fact that we do not have a large sample of sources for which there are mass measurements for the compact objects. This hampers the ability to design statistical studies that simply define groups on the basis of mass. The primary limitation is the small number of NS classifications with dynamical mass measurements. One can work around this problem by classifying sources according to the current paradigms and investigating whether their behavior is self-consistent with their classification. We adopt this strategy to investigate accreting compact objects in terms of X-ray burst production. The selection of some kind of complete population of X-ray binary systems is then very useful, since this can help to limit selection biases that may intrude upon the effort to fairly represent each compact-object class.

We use the archive of the $R X T E$ ASM 
(Levine et al. 1996) over the time interval 1996 January to 2005 January to define a sample of $\mathrm{X}$-ray transients whose outbursts are associated with the disk instability mechanism. In these sources, matter from the companion star slowly fills a quiescent accretion disk until a critical density is reached, and mass then flows to the compact object during an interval of roughly 1-30 months (Chen et al. 1997). X-ray transients are selected because they systematically sweep through a large range in the mass accretion rate, which is one of the most important variables that affects the burst rate for a compact object of a given mass.

All of the sources in the ASM archive are considered, except for those with extragalactic classifications. We use the sum band light curves $(2-12$ $\mathrm{keV}$ ), binned in 1-day time intervals, and we determine three quantities: the maximum weightedaverage flux during any 7-day interval $\left(f_{\max }\right)$, the highest number of consecutive 7-day intervals when the source remains detected above a $5 \sigma$ threshold $\left(N_{\text {det }}\right)$, and the maximum number of consecutive 7-day intervals when the source is below the detection threshold $\left(N_{\text {off }}\right)$. We then apply an ad hoc definition of an X-ray transient as a source with $3 \leq N_{\text {det }} \leq 150$ and $N_{\text {off }} \geq$ 30. The selection criteria are designed to avoid both fast X-ray transients (e.g. XTE J0421+560 and XTE J1901+031) and quasi-persistent sources (e.g. KS 1731-260 and XTE J1716-389). To select a roughly complete sample, while avoiding all of the systematic complications near the ASM detection threshold, we further choose a sample with $f_{\max }>3.0 \mathrm{ASM} \mathrm{c} / \mathrm{s}$, i.e., transients with at least one week above $40 \mathrm{mCrab}$ or $1.0 \times 10^{-9} \mathrm{erg} \mathrm{cm}^{-2}$ $\mathrm{s}^{-1}$ at $2-10 \mathrm{keV}$. This process yields $50 \mathrm{X}$-ray sources. The completeness threshold is estimated from two considerations. First, the ASM weekly sky maps have been used to discover transients (e.g. XTE J1837+037 and XTE J0921-319) down to $25 \mathrm{mCrab}$ (Galloway et al. 2002). Second, the PCA scans of the Galactic Center region provide monitoring functions down to a few mCrab, albeit with only weekly or bi-weekly sampling. The PCA scans decrease the probability that an X-ray transient may go unnoticed in the celestial area where the ASM detection threshold is highest, due to the large number of bright sources.

The list of 50 classical X-ray transients contains 9 BHBs and $10 \mathrm{BHCs}$
(McClintock \& Remillard 2006; Orosz et al. 2004; Casares et al. 2004), 13 X-ray pulsars, 14 known bursters, and 4 sources that remain unclassified. The X-ray pulsars (10 classical ones and 3 X-ray msec pulsars) are excluded from our burst-rate analyses, and the remaining 37 sources are listed in Table 1. The classical X-ray pulsars are excluded because their strong magnetic field focuses the accretion geometry onto the magnetic polar caps, where steady nuclear burning suppresses bursts. The msec X-ray pulsars are known sources of Xray bursts (Strohmayer et al. 2003). However, they may differ from common bursters because of magnetic field effects and/or the chemical composition of accreted matter, since the companions are extremely evolved stars or stripped cores.

Our list of $37 \mathrm{X}$-ray transients consists of four groups: BHBs, BHCs, NS-bursters, and "type unknown". Table 1 is organized in terms of these groups. We note that 5 of the 14 systems in the NS group do not produce bursts in this investigation (col. 10 of Table 1). However, NS classifications can be made with contemporaneous instruments, e.g. Beppo-SAX, or with other instruments during previous outbursts. In particular, X-ray bursts have been recorded from X1711-339 (Cornelisse et al. 2002), SAX J1747.0-2853 (Werner et al. 2004), X1744361 (Emelyanov et al. 2001), X1803-245

(Muller et al. 1998; Wijnands \& van der Klis 1999), and SAX J1810.8-2609 (Natalucci et al. 2000).

Models for X-ray bursts provide predictions of burst rates as a function of the mass accretion rate, the compact object mass, and a set of trial assumptions which are discussed in $\S 4$. Predictions can be tied to observations if it is assumed that the accretion rate scales with the bolometric luminosity, which can be calculated from spectral parameters when there are estimates for the distance and the binary inclination angle (used for the thermal luminosity from the accretion disk). Table 1 lists the values for distance and inclination angle adopted for each source. The $\mathrm{BH}$ masses and distances are taken from McClintock \& Remillard (2006), while we use an average mass for the BHC and NS groups. Distance estimates for the NS group are primarily derived from published analyses of radius-expansion bursts (Strohmayer \& Bildsten 2006). We assume a distance of $8.5 \mathrm{kpc}$ for sources with no published 
distance estimate, except for X1803-245, where a smaller distance $(7.6 \mathrm{kpc})$ is assumed to keep the maximum bolometric luminosity below the Eddington limit.

\subsection{PCA Pointed Observations}

For each of the 37 sources listed in Table 1, we searched the RXTE archive for pointed observations with the PCA instrument (Jahoda et al. 1996). The number of pointings (excluding raster scans) and the total exposure time are listed in cols. 2 and 3 , respectively. We analyzed all of the data that were publicly available, 1996 January through 2004 November for BHs and through 2004 March for NSs. We constructed light curves at $1 \mathrm{~s}$ time resolution for the full bandwidth of the PCA instrument (effectively $2-40 \mathrm{keV}$ ), averaged over the number of operating PCUs. Standard filters were applied to screen out data affected by pointing anomalies, Earth occultations, detector problems, or unusually high rates in the particle background. We further impose a lower limit of $2 \mathrm{mCrab}(5 \mathrm{c}$ $\mathrm{s}^{-1} \mathrm{PCU}^{-1}$ ) on the net flux from the $\mathrm{X}$-ray source to ensure that the source is not in a quiescent state. The latter step excludes many observations of GX339-4.

Two types of PCA spectral analyses are performed for this study. The first task is to evaluate the source luminosity for each observation, in the context of searching for bursts as a function of bolometric luminosity. For this purpose we use PCA "standard 2" data, where the X-ray spectrum from each PCU is accumulated in 129 energy channels with $16 \mathrm{~s}$ time resolution. We use the prescribed methods for background subtraction and the creation of response files, which are never offset from the time of each observation by more than 20 days. Spectral fits are performed with the XSPEC package provided by NASA. The fits are confined to the energy range of 2.9-25.0 $\mathrm{keV}$, and we impose a $1 \%$ systematic uncertainty on each spectral bin. We focus the analyses on PCU \#2 because of its frequent use and excellent calibration, as judged from spectral modeling of observations of the Crab Nebula. Results from PCU \#0 are used in substitution when needed.

Each data interval (excluding bursts, when present) is fit to a spectral model consisting of a disk blackbody, a power-law component, and a Fe $\mathrm{K} \alpha$ emission line. Photoelectric absorption in the ISM is also included in the model; the column density is fixed for each source, and the values are given in Table 1. For the NS group, the Fe line is modeled with a Gaussian profile, while a relativistically broadened line (Laor profile) is used for the $\mathrm{BH}$ and $\mathrm{BHC}$ groups. In addition, a reflection component is needed for BHBs $4 \mathrm{U}$ 1354-64, $4 \mathrm{U}$ 1543-47, and GX339-4. A smeared absorption edge is included in the model for GRO J1655-40 and XTE J1550-564 (Sobczak et al. 2000). To limit systematic problems beyond the range of the PCA sensitivity, we impose a lower limit of $0.5 \mathrm{keV}$ for the disk temperature. Finally, for those data intervals where the minimized value of reduced $\chi^{2}$ remains above 1.3 , we substitute a broken powerlaw model in place of the power law, and we accept the revised fit if there is significant improvement in $\chi^{2}$, while the break energy is above $5 \mathrm{keV}$, so that the break is not convolved with the thermal component.

The second type of PCA spectral analysis is the effort to model the spectra of candidate bursts. The events located in burst searches (see below) are brief, compared to the $16 \mathrm{~s}$ quanta of standard 2 data, and their analysis requires access to the discretionary fast-timing modes allocated to each $R X T E$ observer. Most NS observations include an event mode configuration with 64 energy channels and a time resolution of $125 \mu \mathrm{s}$ or better. There is greater variety in the user modes for BHBs and $\mathrm{BHCs}$; the time resolution is almost always better than $8 \mathrm{~ms}$, but the number of energy channels is more commonly 8 or 16 . For each burst candidate, we integrate the excess flux above the local burst "background", which includes the persistent source emission plus the various types of celestial and detector backgrounds. The net spectrum is then modeled with a blackbody function and photoelectric absorption to determine whether the event is consistent with thermal emission that is expected from a thermonuclear explosion. The mechanics of the fit resemble the procedures used for a source's persistent emission, albeit with fewer PCA energy channels.

The accumulated time of analyzed PCA data for each source is given in col. 4 of Table 1. By group, the total exposures are $3.8 \mathrm{Ms}$ for BHBs, 2.7 Ms for BHCs, and $3.7 \mathrm{Ms}$ for NSs. The 4 sources of unknown type are ignored in our calculations of burst rates, but their total archival time 
is only $0.14 \mathrm{Ms}$, or $1.3 \%$ of the time utilized for burst searches. We conclude that the archive of RXTE pointed observations of our 37 non-pulsing Galactic X-ray transients is highly concentrated into the source classes where measured burst rates can be effectively compared to physical models.

\section{X-ray Timing and Spectral Analyses}

\subsection{Candidate Bursts from PCA Light Curves}

We conduct computer searches for X-ray bursts using the $1 \mathrm{~s}$ light curves derived from the PCA instrument. A candidate burst is defined via variance analysis in the following way. For each $1 \mathrm{~s}$ time bin under scrutiny, we define two background intervals with relative time offsets: $-280<B G 1<-20$, and $180<B G 2<280$. These "background" intervals contain flux contributions from the source's persistent emission, the diffuse X-ray background, and the detector background. The asymmetric gap between these background intervals is designed to contain X-ray bursts, which have rise times of a few seconds and decay times ranging from $10 \mathrm{~s}$ to a couple of minutes (Strohmayer \& Bildsten 2006).

For each background region, we compute the mean and the sample standard deviation: $b_{1}, \sigma_{1}, b_{2}, \sigma_{2}$, respectively. At a particular time $t_{0}$, the count rate $C\left(t_{0}\right)$ is tested for a condition of excess X-ray flux:

$$
\left(C\left(t_{0}\right)-b_{1}\right)>5 \sigma_{1} ;\left(C\left(t_{0}\right)-b_{2}\right)>5 \sigma_{2}
$$

Sample light curves containing X-ray bursts and the offset background regions for burst searches are shown in Fig. 1. Both of these bursts arise from Aql X-1, and they have durations that are significantly shorter and longer, respectively, than the average for that source.

The use of two background regions dampens the rate of false candidates when the source exhibits secular changes in X-ray flux or state transitions. If the available data in a background region are less than $10 \mathrm{~s}$ because of data gaps, then $b$ and $\sigma$ are set to zero, effectively removing one side of the variance test (Eq. 1). The use of sample standard deviations, rather than Poisson statistics, is required because there is considerable source flickering as is evident in the power density spectra of X-ray binaries at $1 \mathrm{~Hz}$ (van der Klis 2006; McClintock \& Remillard 2006).

There are two noteworthy details to report regarding the effort to uniformly search for burst candidates using PCA light curves and Eq. 1. First, the NS source X1658-298 exhibits X-ray eclipses and absorption dips (Wijnands et al. 2002) that complicate the variance analyses. This requires hands-on attention to the light curves, and we added one burst candidate that was not recognized by the automatic search process. Secondly, the NS system EXO 1745-248 sometimes exhibits extraordinary light curves in the form of structured dips that mingle with X-ray bursts (Markwardt et al. 2000; Wijnands et al. 2005). During such episodes, the variances in the background regions can become very large, given the mean count rate. However, upon review of the results, we choose to accept the automatic search results for this source.

A burst candidate is defined with one additional free parameter, which is how many consecutive seconds, $j$, that Eq. 1 is true. To investigate this parameter, we have iterated the search for burst candidates, varying $j$ over the range $3-13$ s. The results are shown in Fig. 2. The rates for finding burst candidates are shown separately for the NS group (triangles; excluding the eclipsing source X1658-298) and for the combined BHB and $\mathrm{BHC}$ groups (squares). For the NS group, the rate is high and the distribution is flat versus $j$. Lower rates are seen for $\mathrm{BHs}$, and the rate decreases more steeply with $j$, as high-amplitude examples of fast flickering transition to events with longer time scales that might be X-ray bursts.

Burst searches should be as complete as possible in order to fairly compare the burst rates with physical models for thermonuclear explosions. For the NS group, the number of burst candidates is insensitive to the choice of $j$ (Fig. 2). We therefore adopt $j=7$ as a choice that effectively avoids many fast events for BHBs and BHCs, while preserving the need to implement search criteria that are as short as the timescale for decay to half-maximum in true bursts (Strohmayer \& Bildsten 2006). This decision is not expected to create a bias against detection true bursts in $\mathrm{BH}$ systems, since our theoretical models $(\S 4)$ show that burst durations 
are longer for heavy compact objects as compared to NS bursts. The number of burst candidates for each source with $j=7$ is included in Table 1 .

Before moving on to the method to distinguish true X-ray bursts from candidates, we examine whether the selection of candidates appears to be systematically affected by the search parameters. Some of the burst-search criteria are scientifically motivated, e.g. the window function for variance analysis. Other choices (e.g. the $5 \sigma$ threshold) are dictated by statistics and affected by non-burst source behavior. It is therefore instructive to compare the detectability of the burst candidates versus the detection threshold. As the search window steps over a given burst, the critical value in determining the condition of excess $\mathrm{X}$-ray flux is the $j$ th brightest point, evaluated in units of signal-to-noise relative to the background region that imposes the highest threshold. We can use this quantity as a detectability parameter: $D_{j}=\operatorname{maximum}\left\{x_{j}\left(t_{i}\right)\right\}$, where $t_{i}$ is incremented over the entire burst candidate, $x_{j}(t)=$ minimum value of $\{r(t), r(t+1), \ldots r(t+j-1)\}$, $r(t+n)=\left(C(t+n)-b_{k}\right) / \sigma_{k}$, and the index $k$ is the one (of two) background region that minimizes $r(t+n)$.

The detectability results for burst candidates $(j=7 \mathrm{~s})$ are shown in Fig. 3 . For the NS group we use all of the burst candidates tabulated in Table 1 except for those from X1658-298. The large majority of NS burst candidates have detectability that is well above the $5 \sigma$ threshold; $86 \%$ have detectability above $10 \sigma$ and $72 \%$ are above $30 \sigma$. On the other hand, the detectability distribution for the BHB and BHC groups lies closer to the detection threshold, with only $39 \%$ above $10 \sigma$. Therefore, at the burst candidate level, we already see a large difference between the rates and statistical properties of results for the NS group as compared to $\mathrm{BHBs}$ and BHCs.

\subsection{Burst Confirmation from PCA Spec- tral Analyses}

The standard method to identify a burst candidate as a bona fide type I X-ray burst (i.e. a thermonuclear explosion) is to demonstrate that the X-ray flux above the persistent level has a thermal (blackbody) spectrum (Strohmayer \& Bildsten 2006) with a temperature below $3 \mathrm{keV}$. The large majority of PCA obser- vations of the sources in the NS group provide data telemetry in an event mode with 64 or more spectral channels and $122 \mu$ s or better time resolution, and this facilitates the effort to isolate the burst candidate and conduct meaningful spectral analyses. For all of the burst candidates that we detect (Table 1, col. 9) we integrate the excess flux and fit the net spectrum to a blackbody model, as described in $\S 2.2$. All but two of the candidate bursts from the NS group (i.e., 135 of 137) are confirmed as type I X-ray bursts. We further note that all of these bursts exhibit smooth temporal profiles that include a fast rise and slower decay, as illustrated in Fig. 1, except for some bursts from EXO 1745-248 and X1658-298 that are co-mingled with structured absorption dips.

The 20 burst candidates for BHBs and BHCs are significantly fewer than the NS candidates, while their temporal profiles are substantially more complicated. We divide them into two groups in the effort to assess their viability as thermonuclear explosions from the surface of a compact object. None of the $\mathrm{BH}$ and BHC burst candidates has profiles that resemble the type I bursts shown in Fig. 1. Nevertheless, we assess them primarily on the basis of spectral properties, as done for the NS burst candidates.

In 15 cases, the flare timescale (FWHM) is 30$100 \mathrm{~s}$, and this provides access to at least one 16-s spectrum from PCA standard 2 mode (129 energy bins) during the peak of the event. Some illustrations of these $\mathrm{BH}$ burst candidates are given in Fig. 4. For the majority (9) of long-duration burst candidates, the trigger occurs near the beginning or the end of the observation, when there is only one background region available in the burst search process. Two such examples are shown in the lowest two panels of Fig. 4. Given the chronic drifts in the X-ray flux of $\mathrm{BH}$ transients, it seems obvious that such events are spurious candidates, selected when a strong local maximum happens to occur near the beginning or end of an observation. The remaining seven burst candidates with long durations are perhaps the most interesting, since most of them occur during bright X-ray states, when heavy compact objects are expected to burst most frequently (see $\S 5$ ). Four illustrative examples are shown in the upper panels of Fig. 4.

For each of the 15 long-duration burst candidates, we subtracted the spectrum of the persis- 
tent emission from the burst maximum, as sampled in the $16 \mathrm{~s}$ quanta of the standard 2 data mode. We then fit the results to spectral models, as we had done for NS burst candidates. None of these burst candidate spectra is consistent with a blackbody spectrum. In most cases the spectral model requires both a thermal and a power-law component, suggesting a simple flaring of the persistent emission. Some of the burst candidates, e.g. those from XTE J1550-564, are well fit as simple power-law spectra with photon indices in the range 2.8-3.7.

The remaining $5 \mathrm{BH}$ and $\mathrm{BHC}$ burst candidates are fast events, and four examples are displayed in Fig. 5. Three candidates occur in faint X-ray states (persistent count rates $<100 \mathrm{c} \mathrm{s}^{-1} \mathrm{PCU}^{-1}$ ). The two fast events in bright states are both from $4 \mathrm{U} 1630-47$, and one of these is displayed in the top-right panel of Fig. 5. The spectral analyses of the fast events must depend on the discretionary telemetry modes that have much higher time resolution than the standard 2 mode. In these particular cases, the selected modes provide 24 to $40 \mathrm{PCA}$ energy channels over the range of 2 to $40 \mathrm{keV}$. For the short duration candidates, we again find that the burst spectra are inconsistent with a blackbody function and a temperature below $3 \mathrm{keV}$. The results for the faint burst from XTE J1650500 (see Fig. 5) are marginal. However, Tomsick et al. (2003) conducted a detailed study of flares from this source during the faint decay of its outburst, and they concluded that those events are not type I X-ray bursts.

Finally, we note that one can construct a source hardness ratio (after subtracting the background) from using the PCA count rate at $15-40 \mathrm{keV}$ relative to the rate at $2-15 \mathrm{keV}$. Since thermal spectra (1-3 keV) contribute negligible flux above $15 \mathrm{keV}$, the plot of this hardness ratio shows a dip during type I X-ray bursts, while other types of flares in our study appear either neutral or show increases at times near the burst trigger.

We conclude that we are unable to find any type I X-ray bursts during $6.5 \mathrm{Ms}$ of PCA exposures of BHBs and BHCs. It remains to be seen (§5.2) whether the exposure times, binned versus Eddington luminosity, are sufficient to violate the predictions of the burst model for massive compact objects with a hard surface.

\subsection{X-ray Spectral Differences between Neutron Star and Black Hole Groups}

X-ray spectral fits ( $\$ 2.2)$ are necessary to compute the bolometric luminosity for each observation (excluding any bursts), since this quantity serves as a proxy for the local accretion rate that is used to derive the burst rate function ( $\$ 5.1)$. This large set of spectral parameters also allows us to compare different source classifications, with a desire to gain corroborative evidence for intrinsic differences that would help to justify the group assignments gained from the literature.

We examined the distributions in the value of the power-law index for the NS and BH groups, and we find that they are similar. For each group, a steep power-law component is seen during soft $\mathrm{X}$-ray states, with photon index, $\Gamma>2.5$. The less common hard state shows a flatter power-law component with $\Gamma \sim 1.7$. Such results are typical for BHBs (McClintock \& Remillard 2006), and also for the hard and soft branches of atoll-type NS systems (Muno et al. 2003; Maitra \& Bailyn 2004).

However, the temperature distributions for the thermal component appear to effectively distinguish the $\mathrm{BH}$ and NS groups. These measurements are confined to the soft states, since the thermal component is generally not visible in PCA data (i.e., above $2 \mathrm{keV}$ ) in the hard states of either BHBs (McClintock \& Remillard 2006) or NSs (e.g., Gierliński \& Done 2002) . This is shown in Fig. 6. The $\sim 1 \mathrm{keV}$ temperatures for BHs (blue line) and BHCs (green line) are clearly disjoint from the $\sim 2 \mathrm{keV}$ temperature of NS systems. We note that the NS histogram in Fig. 6 excludes the results from two dipping sources (X1658-298 and EXO 1745-248), where the temperature distributions may be affected by variations in the absorption column density. We also confined the results for BHs and BHCs to observations in the thermal state (McClintock \& Remillard 2006), ignoring the observations in the "steep power law", a spectral state that is not observed for NS systems.

The large majority of X-ray transients do exhibit soft X-ray states. Exceptions in our sample are limited to two BHBs (XTEJ 1118+480 and $4 \mathrm{U}$ 1354-64), where the outbursts are confined to the hard state. We conclude that the group assignments of Table 1 can be reproduced with the combined use of dynamical mass measurements and 
the empirical conclusion that heavy compact objects in the thermal state exhibit mean temperatures below $1.5 \mathrm{keV}$. Alternatively, sources with mean soft-state temperatures above $1.5 \mathrm{keV}$ are all associated with the NS group (including the two dippers). These results provide evidence for intrinsic differences between the $\mathrm{BH}$ and NS groups that are independent of the phenomenon of $\mathrm{X}$ ray bursts. The design of our statistical study is thereby strengthened.

\section{The X-ray Burst Model}

We describe the burst model that is used to calculate the rates of type I X-ray bursts for both accreting NSs and heavy compact objects. In $\S 5$, we compare the model predictions to the measured NS burst rate function and the upper limits derived for $\mathrm{BHBs}$ and $\mathrm{BHCs}$. We use the theoretical model of Cooper \& Narayan (2005), which is an expanded and improved version of the model of Narayan \& Heyl (2003). In the subsections below, we summarize the assumptions of the model (Cooper \& Narayan 2005), and we describe the modifications to the model that are relevant to the present study.

\subsection{Modifications to the Burst Model for Neutron Stars}

We assume that gas accretes spherically onto a NS of gravitational mass $1.4 M_{\odot}$ and areal radius $10.4 \mathrm{~km}$. The accreted gas is composed of $70 \%$ hydrogen, $28 \%$ helium, $1.6 \%$ CNO elements, and $0.4 \%$ iron by mass. We assume that the crust either forms an ordered lattice at high densities or is amorphous. Furthermore, we assume that the stellar core emits neutrinos via either modified Urca reactions or pionic reactions.

The only difference between the superburst code described by Cooper \& Narayan (2005) and the model used here to calculate hydrogen- and helium-triggered bursts is the way in which we calculate the inner temperature boundary condition. The long recurrence times of superbursts $(\sim$ years to decades) imply that the thermal diffusion depth is very deep into the NS, often deeper than the crust-core interface. Therefore, to solve for the thermal and hydrostatic profiles of the stellar crust, Cooper \& Narayan (2005) integrated down to the crust-core interface, at which they applied an inner temperature boundary condition. However, hydrogen- and helium-triggered burst recurrence times are much shorter, so the thermal diffusion depth is much closer to the stellar surface. Narayan \& Heyl (2003) integrated only to this diffusion depth, at which they applied their inner temperature boundary condition. Since they were unable to model the crust of the star below neutron drip, they assumed that the star was isothermal below the diffusion depth. The newer code (Cooper \& Narayan 2005) accurately models the entire crust, rendering this assumption unnecessary.

In an X-ray transient, the time-averaged accretion rate prior to the transient outburst, $\langle\dot{M}\rangle$, is much lower than the accretion rate during the burst, $\dot{M}_{\text {burst }}$. The energy generation rates due to thermonuclear burning, compressional heating, and deep crustal heating, which help set the thermal profile of the crust, are all proportional to the accretion rate. Since the diffusion time down to the crust-core interface in a NS is $\sim 10-100$ years, the thermal profile of most of the crust will be set by the time-averaged accretion rate $\langle\dot{M}\rangle$, not the current accretion rate $\dot{M}_{\text {burst }}$ during the burst. We allow for this as follows in the calculations.

To calculate the inner temperature boundary condition for NS transients, applied at the diffusion depth $\Sigma_{\text {diff, }}$, we first solve for the timeaveraged thermal profile of the NS star crust. Using the time-averaged accretion rate $\langle\dot{M}\rangle$, we integrate down to the crust-core interface and apply the procedure described by Cooper \& Narayan (2005) to calculate the inner temperature boundary condition at this interface. The resulting equilibrium configuration gives a temperature profile as a function of depth, $T(\Sigma)$. We then use the burst accretion rate $\dot{M}_{\text {burst }}$ and proceed with the burst calculation as described by Narayan \& Heyl (2003), setting the inner temperature boundary condition to the value of the temperature profile $T(\Sigma)$ evaluated at the diffusion depth $\Sigma_{\text {diff }}$.

\subsection{Modifications to the Burst Model for Black Holes}

In our black hole burst model, we assume that BHs are compact objects with solid surfaces. We assume spherical accretion and the same gas composition as that used in the NS model. We assume a gravitational mass of $M=8.0 M_{\odot}$, 
and we choose three stellar areal radii, $R=$ $9 / 8 R_{\mathrm{S}}, 2 R_{\mathrm{S}}$, and $3 R_{\mathrm{S}}$, where $R_{\mathrm{S}}=2 G M / c^{2}$ is the Schwarzschild radius. Note that $R=9 / 8 R_{\mathrm{S}}$ is the physical lower limit on the radius of a compact object with a solid surface and a nonpositive density gradient (Buchdahl 1959). The maximum stellar radius is not well constrained, but X-ray observations of BHBs imply that the inner radius of the accretion disk is $\lesssim 3 R_{\mathrm{S}}$ (Davis et al. 2005; Li et al. 2005; Shafee et al. 2006). Furthermore, we assume that the outermost layer of the compact object consists of normal baryonic matter. Below this layer we make no assumptions regarding either the stellar structure or composition. We define the transition rest mass density $\rho_{0 \text {,trans }}$ to be the density that separates these two regimes. Thus for $\rho_{0}<\rho_{0}$,trans the structure and composition are that of a NS and for $\rho_{0}>\rho_{0, \text { trans }}$ the structure and composition are unknown.

The BH burst calculation is identical to the NS burst calculation, with three exceptions: (i) To solve for the time-averaged thermal profile, we integrate into the compact object until the rest mass density $\rho_{0}=\rho_{0 \text {,trans, }}$, whereas in the NS case we integrate to the crust-core interface, where $\rho_{0}=2 \times 10^{14} \mathrm{~g} \mathrm{~cm}^{-3}$. (ii) We do not know up to what density $\rho_{0, \text { trans }}$ a hypothetical massive compact object with a surface should consist of normal baryonic matter. Thus, we choose three values: $4 \times 10^{10} \mathrm{~g} \mathrm{~cm}^{-3}, 4 \times 10^{11} \mathrm{~g} \mathrm{~cm}^{-3}$, and $4 \times 10^{12}$ $\mathrm{g} \mathrm{cm}^{-3}$. We find that the results of our calculations are quite insensitive to the specific value of $\rho_{0, \text { trans }}$. Therefore, we choose $\rho_{0, \text { trans }}=4 \times 10^{11}$ $\mathrm{g} \mathrm{cm}^{-3}$, the neutron drip point, as our fiducial transition rest mass density. At the depth where $\rho_{0}=\rho_{0, \text { trans }}$, we assign a fixed inner temperature boundary condition $T_{\mathrm{IBC}}$ instead of assuming that the interior cools via either modified Urca reactions or pionic reactions. Since we make no assumptions about the interior of the compact object, we cannot determine the proper temperature at neutron drip self-consistently. Thus, we choose seven values for $T_{\mathrm{IBC}}$, covering the range $10^{5} \mathrm{~K}$ to $10^{8} \mathrm{~K}$ in logarithmic steps of 0.5 . These values should generously bracket the likely true proper temperature. (iii) The thermal diffusion time to the depth at which $\rho_{0}=\rho_{0 \text {,trans }}$ is modeldependent, but it is typically on the order of a month. This is shorter than the timescale over which the accretion rate changes in a $\mathrm{BH}$ tran- sient, which is on the order of several months. Therefore, in our model we treat BH transients as quasi-persistent systems and set $\langle\dot{M}\rangle=\dot{M}_{\text {burst }}$. This is different from the NS case. Nonetheless, we have done $\mathrm{BH}$ burst rate calculations in which $\langle\dot{M}\rangle \ll \dot{M}_{\text {burst }}$, and the results are generally very similar to those presented in this paper.

\section{X-ray Burst Rates and Comparisons with Burst Models}

\subsection{Burst Rates and Model Results for Neutron Stars}

The $R X T E$ satellite is in a low-Earth orbit, where astronomical instruments routinely experience data gaps due to Earth occultations and electronics shutdown during spacecraft passage through the South Atlantic Anomaly. It is therefore highly impractical to directly measure the time between consecutive X-ray bursts from a given source. We therefore adopt a different strategy and statistically consider every continuous data interval as an opportunity to capture X-ray bursts. We record the exposure time and any burst statistics, and we extract and fit the average PCA spectrum (excluding any bursts) with the disk-blackbody + power-law model, as described in $\S 2.2$.

The exposures and confirmed bursts for all of the X-ray sources in each compact object group are then accumulated in logarithmic intervals of the ratio of the bolometric luminosity to the Eddington luminosity. For each data interval, we determine the bolometric luminosity $\left(L_{\mathrm{bol}}\right)$ from the spectral parameters, summing the contributions from the power law, integrated over $1-30 \mathrm{keV}$, and the accretion disk: $L_{d i s k}=1.29 \times 10^{35} N_{d b b}(d / 10$ $\mathrm{kpc})^{2}(T / \mathrm{keV})^{4} / \cos (i) \operatorname{erg~s}^{-1}$, where $N_{d b b}$ and $T$ are the 'diskbb' parameters in XSPEC, and the distance $(d)$ and binary inclination $(i)$ are listed for each source in Table 1. For the Eddington luminosity, we use $L_{\mathrm{Edd}}=1.3 \times 10^{38}\left(M / M_{\odot}\right)$ erg $\mathrm{s}^{-1}$, where the compact object mass $(M)$ is also given in Table 1. We note that 'diskbb' is a nonrelativistic model that is only used to integrate the thermal spectrum from the accretion disk.

We refer to the burst rates, measured in logarithmic intervals of $L_{\mathrm{bol}} / L_{\mathrm{Edd}}$, as the burst rate function. The results for the NS group are shown in Figs. 7 and 8. The error bars $(1 \sigma)$ are calcu- 
lated by assuming Poisson statistics for counting bursts in each bin, and the $90 \%$ confidence interval is shown when no bursts were found in a given bin. Fig. 7 shows the predicted burst rates for four different models of the NS group, all with a gravitational mass of $1.4 M_{\odot}$ and an areal radius of $10.4 \mathrm{~km}$. As explained in $\S 4.1$, these predictions are derived from models tailored to transient Xray sources, where the inner temperature boundary condition for the NS is set by the long-term average accretion rate of $\langle\dot{M}\rangle=10^{-3} \dot{M}_{\text {Edd }}$. Of the four models shown, the "Pion" model with an ordered crust is inconsistent with the data. This model predicts bursts over a very narrow range of $\dot{M}$ ( factor of two), whereas the observations of individual sources indicate a substantial burst rate over at least an order of magnitude range in $\dot{M}$. We may thus rule out this model for NSs, in agreement with previous studies (Narayan \& Heyl 2003; Cooper \& Narayan 2004; Brown 2004; Cooper \& Narayan 2005). The other three models all give nearly the same predictions and are generally consistent with the data. The predicted burst rate of $\sim 5 \times 10^{-5} \mathrm{~s}^{-1}$ agrees very well with the observations, as does the position of the peak as a function of accretion luminosity. This is significant because the model has essentially no free parameters. The range of luminosity over which the burst rate is substantial is $\sim 0.8$ in $\log \left(L_{\mathrm{bol}} / L_{\mathrm{Edd}}\right)$, which is somewhat lower than the observed range, which is $\sim 1.2$. Part of the discrepancy may be due to uncertainties in the observational estimates of $L_{\text {bol }}$ (see below). Fig. 8 shows the effect of the NS radius on the predictions. We see that, over the range $R=8.2-13.1$ $\mathrm{km}$, the models are generally indistinguishable and consistent with the data. Narayan \& Heyl (2003) used their type I X-ray burst models to argue that small radii are preferred. The present calculations, which treat the inner boundary condition more carefully, lead to a different conclusion.

We can roughly characterize the success of the NS burst model in an integral fashion, as follows. We calculate the total number of predicted bursts by considering each NS exposure and multiplying the exposure time by the burst rate given by the model (e.g. mUrca) for the luminosity on that occasion. The predicted total is 102 bursts, while the measured number is 135 . We consider this level of agreement to be remarkably good, given the uncertainties in the true values of the mass accretion rate.

The measured burst rates and the model predictions have similar values, but the model has a sharper profile, peaking near $L_{\mathrm{bol}} / L_{\mathrm{Edd}} \sim 0.2$. To understand the significance of this deviation, we must address the factors of uncertainty in our determinations of the Eddington-scaled luminosity. For statistical uncertainties, we roughly estimate that the most significant terms in the error analysis are a $20 \%$ uncertainty in $M$ and a $30 \%$ uncertainty in $d$, and a $15 \%$ uncertainty in calculating the luminosity from the spectral parameters. This leads to a convolved statistical uncertainty of $65 \%$ in $L_{\mathrm{bol}} / L_{\mathrm{Edd}}$ (noting the quadratic dependence on $d$ ). There are also important systematic uncertainties, especially the ability to estimate the mass accretion rate from the bolometric luminosity. In view of these uncertainties, it is not at all surprising that the measured burst function is broader than the model.

Can the distance uncertainty alone account for the broad shape of the measured burst function? We conducted an exercise to find a set of optimal distances that might bring the measurements into better agreement with the burst model, without altering any other steps in the data analyses. One can view the burst rate measurements, the statistical uncertainties, and the burst model results (e.g. mUrca) in Fig. 7 in terms of a $\chi_{\nu}^{2}$ test with no degrees of freedom. With no considerations of uncertainties along the luminosity axis, we find $\chi_{\nu}^{2}=5.0$. The set of optimized distances for the $13 \mathrm{NSs}$ achieves $\chi_{\nu}^{2}=0.9$, with an average shift in distance of $3 \%$ and an rms shift of $32 \%$. If the broad shape of the burst rate function is, in fact, primarily an effect of distance errors, then the true distances for Aql X1 and $4 \mathrm{U}$ 1608-52, which account for the majority of the NS exposure time, must both be $\sim 47 \%$ larger than the estimates given in Table 1. Such deviations remain within the realm of possibility, since the primary method for distance determinations, which utilizes radius-expansion bursts, has its own imperfections and systematic uncertainties (Galloway et al. 2003). However, we emphasize that this consideration of the distance uncertainty is merely an attempt to explore that topic in a quantitative manner. The results do not imply that other sources of errors should be neglected. 


\subsection{Burst Upper Limits and Model Re- sults for Black Holes}

The measured burst functions for BHB groups are shown in Fig. 9. For each luminosity bin, there are two upper limits that correspond to the $90 \%$ confidence values (assuming Poisson statistics) for the BHB group and the combined BHB and BHC group, respectively. Fig. 9 also shows the predictions of the burst model for heavy compact objects. Like the NS model results, the predicted burst rates for large values of $L_{\mathrm{bol}} / L_{\mathrm{Edd}}$, where the rates are maximized, are quite insensitive to the inner temperature boundary condition. These bursts ignite very near the surface of the compact object, which is thermally insulated from the stellar interior (Cooper et al. 2006), and thus the burst rates are quite insensitive to the inner temperature boundary condition. Bursts at lower accretion rates generally are triggered much deeper in the ocean, where the thermal profile of the ignition region is more highly correlated with that of the inner crust. Consequently, the burst rates are much more sensitive to the inner temperature boundary condition in this regime.

As illustrated in Fig. 9, the results of all $21 \mathrm{BH}$ burst models (i.e. 3 stellar radii $\times 7$ inner temperatures) are grossly inconsistent with the observed upper limits for $L_{\mathrm{bol}} / L_{\mathrm{Edd}} \gtrsim 0.1$. To quantify the degree to which the model results are inconsistent with the data, we proceed as follows. For a particular BH model $\mu$ and assuming Poisson statistics, the probability of observing zero bursts in an accretion rate bin given that the model is an accurate description of BHs found in nature is simply

$$
P_{i}(0 \mid \mu)=\exp \left(-r_{i} t_{i}\right),
$$

where $i$ denotes the accretion rate bin, $r_{i}$ is the burst rate (bursts $\mathrm{s}^{-1}$ ) for bin $i$ calculated using the theoretical model $\mu$, and $t_{i}$ is the total exposure time for bin $i$. The probability of observing zero bursts in all bins given that model $\mu$ is correct is therefore

$$
P(0 \mid \mu)=\prod_{i} P_{i}(0 \mid \mu)=\exp \left(-\sum_{i} r_{i} t_{i}\right) .
$$

We calculate the probability of observing zero bursts given that $\mathrm{BHs}$ have solid surfaces for each of the stellar areal radius and inner temperature boundary condition models discussed in
$\S 4$. The theoretical burst rates are quite sensitive to the interior physics of the compact object for $\log \left(L_{\mathrm{bol}} / L_{\mathrm{Edd}}\right) \leq-1.5$. Therefore, when we calculate the probability of observing zero bursts, we consider only those accretion rate bins $i$ such that $\log \left(L_{\mathrm{bol}} / L_{\mathrm{Edd}}\right) \geq-1.5$. The results are listed in Table 2. The probability that our observational measurements are consistent with the assertion that $\mathrm{BHs}$ are compact objects with solid surfaces is less than $2 \times 10^{-7}$. When we include BHCs, we obtain an even more significant result, viz., the probability is less than $3 \times 10^{-13}$ (see Table 3 ). We are unable to construct any model of a compact object with a solid surface composed of normal baryonic matter that is compatible with observations.

\section{Discussion and Conclusions}

The absence of type I X-ray bursts in BHBs and BHCs fully supports the current paradigm: black holes do not have a solid surface that can accumulate accreting gas and produce the recurrent nuclear explosions that characterize accretion onto NSs. This study confirms the argument proposed by Narayan \& Heyl (2002) and the subsequent analysis of T03 that the absence of type I X-ray bursts suggests the presence of a $\mathrm{BH}$ event horizon. In the present paper, we significantly improve the quantification of the discrepancy between the upper limits for $\mathrm{BH}$ bursts and the model predictions for heavy compact objects with a hypothesized hard surface. This is done by choosing sources that spend appreciable time in states of high luminosity, by explicitly measuring the burst rate as a function of the estimated accretion rate, and by making improvements to the burst model. The luminosity-binned exposure times of the $R X T E$ sample of X-ray transients are sufficient to categorically exclude all models of heavy compact objects with radii and inner temperatures within the possible ranges for heavy compact objects found in nature, as long as the accretion conditions conform to the general assumptions of the burst model. By combining the luminosity-binned exposure times with the results of our burst model, we calculate the probability that BHBs contain heavy compact objects with solid surfaces to be less than $2 \times 10^{-7}$. For the combined BHB and BHC group, the probability drops to less than $3 \times 10^{-13}$. 
Our compilation of spectral fit parameters allows us to compare $\mathrm{NS}$ and $\mathrm{BH}$ groups in terms of properties that have nothing to do with X-ray bursts. Systematic differences are found in the temperatures of the thermal component that is observed during soft X-ray states (Fig. 6). The hotter characteristic temperatures of NS systems is likely to originate in the boundary layer that forms when accreting matter reaches the NS surface. This is another consequence of the NS hard surface that is being investigated for implications of BH event horizons (Done \& Gierliński 2003), as noted in the $\S 1$. In the context of our X-ray burst study, we confirm the kinship between dynamical $\mathrm{BHBs}$ and $\mathrm{BHC}$, as well as the separate identity of all the known bursters.

The burst model performs well in predicting the number of type I bursts in our sample of NS transients. The results agree reasonably well with the burst rates $\sim 7.7 \times 10^{-5} \mathrm{~s}^{-1}$ measured by Cornelisse et al. (2003) with the Beppo-SAX Wide Field Camera for three active sources. Our measured burst rate function is broader than the model predictions, but significant broadening is expected due to the uncertainties in the distance to each binary system. Systematic uncertainties may further contribute to any discrepancies. The burst rate depends, in part, on the mass accretion rate, which we calculate from a simple scaling of the bolometric luminosity. While this process appears to be reasonable for X-ray states dominated by a thermal spectrum, it is uncertain whether the non-thermal states operate with similar radiative efficiency. This includes the hard (steady jet) and "steep power-law" states of BH systems (McClintock \& Remillard 2006), and an analogous hard state for NS transients, as well. However, we note that the exposure times for each type of compact object are dominated by the soft $\mathrm{X}$-ray states where thermal radiation contributes most of the emitted energy. This is especially true at high luminosity, where the $\mathrm{BH}$ groups display gross inconsistencies between the model burst rates and the observed upper limits for thermonuclear explosions. Thus, the uncertainties in the mass accretion rate do not seriously weaken our conclusion that BHs lack a solid surface.

Finally, one must question whether our modeling efforts for the hypothesized massive compact objects cover all of the options available to nature. Our results seriously restrict the possibility that black holes have a surface composed of normal baryonic matter. However, the results do not constrain the viability of more exotic $\mathrm{BH}$ models (e.g. Chapline et al. 2001; Robertson \& Leiter 2002; 2003; Abramowicz, Kluzniak, \& Lasota 2002, Yuan, Narayan, \& Rees 2004). There are similar concerns for our assumption of a spherical accretion geometry, and the implicit presumption that any accretion-focusing effects (e.g. magnetic field) would cause observable pulsations (for objects with surfaces) that are not observed for BHBs and BHCs. As noted in $\S 1$, the best strategy remains to search for solid surface effects in BHBs with as many techniques as possible. Nevertheless, we have shown in this paper that type I X-ray bursts provide strong indirect evidence for $\mathrm{BH}$ event horizons, and it would appear that the evidence can be refuted only by invoking rather exotic physics.

Partial support was provided by the NASA contract to MIT for RXTE instruments, and NASA grant NNG04GL38G to R. N.

\section{REFERENCES}

Abramowicz, M. A., Kluzniak, W., \& Lasota, J.-P. 2002, A\&A, 396, L31

Brown, E. F. 2004, ApJ, 614, L57

Buchdahl, H. A. 1959, Physical Review , 116, 1027

Casares, J., Zurita, C., Shahbaz, T., Charles, P. A., \& Fender, R. P. 2004, ApJ, 613, L133

Chapline, G. et al. 2001, Philos. Mag., 81, 235

Charles, P. A. \& Coe, M. J. 2006, in "Compact Stellar X-ray Sources," eds. W. H. G. Lewin \& M. van der Klis, (Cambridge: Cambridge U. Press), 215

Chen, W., Shrader, C. R., \& Livio, M. 1997, ApJ, 491,312

Cooper, R. L., Mukhopadhyay, B., Steeghs, D., \& Narayan, R. 2006, ApJ, in press; astro$\mathrm{ph} / 0508194$

Cooper, R. L., \& Narayan, R. 2004, AAS/High Energy Astrophysics Division, 8, \#17.28 
Cooper, R. L., \& Narayan, R. 2005, ApJ, 629, 422

Cornelisse, R., et al., 2002, A\&A, 392, 885

Cornelisse, R., et al. 2003, A\&A, 405, 1033

Cumming, A. \& Bildsten, L. 2000, ApJ, 544, 453

Davis, S. W., Blaes, O. M., Hubeny, I., \& Turner, N. J. 2005, ApJ, 621, 372

Done, C., \& Gierliński, M. 2003, MNRAS, 342, 1041

Emelyanov, A. N., Aref'ev, V. A., Churazov, E. M., Gilfanov, M. R., \& Sunyaev, R. A. 2001, AstL, 27, 781

Fender, R., Gallo, E., \& Jonker, P. G. 2004, MNRAS, 343, L99

Fujimoto, M. Y., Hanawa, T., \& Miyaji, S. 1981, ApJ, 247, 267

Fushiki, I., \& Lamb, D. Q. 1987, ApJ, 317, 368

Galloway, D. K., Chakrabarty, D., Morgan, E. H., \& Remillard, R. A. 2002, ApJ, 576, L137

Galloway, D. K., Psaltis, D., Chakrabarty, D., \& Muno, M. P. 2003, ApJ, 590, 999

Garcia, M. R., McClintock, J. E., Narayan, R., Callanan, P., Barret, D., \& Murray, S. S. 2001, ApJ, 553, L47

Gierliński, M., \& Done, C. 2002, MNRAS, 337, 1373

Igumenshchev, I. V., Narayan, R., \& Abramowicz, M. A. 2003, ApJ, 592, 1042

Jahoda, K., Swank, J. H., Giles, A. B., Stark, M. J., Strohmayer, T., Zhang, W., \& Morgan, E. H. 1996, Proc. SPIE, 2808, 59

Jonker, P. G., Bassa, C. G., Nelemans, G., Juett, A. M., Brown, E. F., \& Chakrabarty, D. 2006, MNRAS, in press; astro-ph/0602625

Kalogera, V., \& Baym, G. 1996, ApJ, 470, 61

Kuulkers, E., Homan, J., van der Klis, M., Lewin, W. H. G., \& Mendez, M. 2002, A\&A, 382, 947

Levine, A. M., et al. 1996, ApJ, 469, L33
Li, L.-X., Zimmerman, E. R., Narayan, R., \& McClintock, J. E. 2005, ApJS, 157, 335

Maitra, D., \& Bailyn, C. D. 2004, ApJ, 608, 444

Markwardt, C. B., Strohmayer, T. E. , Swank, J. H. \& Zhang, W. 2000, IAUC 7482

McClintock, J. E, Narayan, R., \& Rybicki, G. B. 2004, ApJ, 615, 402

McClintock, J. E. \& Remillard, R. A. 2006, in "Compact Stellar X-ray Sources," eds. W. H. G. Lewin \& M. van der Klis, (Cambridge: Cambridge U. Press), 157

Menou, K., Esin, A. A., Narayan, R., Garcia, M. R., Lasota, J.-P., \& McClintock, J. E. 1999 ApJ, 520, 276

Muller, J. M., Smith, M. J. S., D'Andreta, G., \& Bazzano, A. 1998, IAUC, 6867

Muno, M. P., Remillard, R. A., \& Chakrabarty, D. 2003, ApJ, 568, L35

Narayan, R. 2003, A\&G, 44, 6.22; astro$\mathrm{ph} / 0310692$

Narayan, R., \& Heyl, J. S. 2002, ApJ, 574, L139

Narayan, R., \& Heyl, J. S. 2003, ApJ, 599, 419

Narayan, R., Igumenshchev, I. V., \& Abramowicz, M. A. 2000, ApJ, 539, 798

Narayan, R., Garcia, M. R., \& McClintock, J. E. 1997, ApJ, 478, L79

Narayan, R., \& Quataert, E. 2005, Science, 307, 77

Narayan, R., \& Yi, I. 1995, ApJ, 444, 231

Natalucci, L, Bazzano, A., Cocchi, M., Ubertini, P., Heise, J., Kuulkers, E., in't Zand, J. J. M., \& Smith, M. J. S. 2000, ApJ, 536, 891

Orosz, J. A., McClintock, J. E., Remillard, R. A., \& Corbel, S. 2004, ApJ, 616, 376

Paczynski, B. 1983, ApJ, 264, 282

Quataert, E., \& Gruzinov, A. 2000, ApJ, 539, 809

Robertson, S. L., \& Leiter, D. J. 2002, ApJ, 565, 447 
Robertson, S. L., \& Leiter, D. J. 2003, ApJ, 596, L203

Shafee, R., McClintock, J. E., Narayan, R., LiXin, L., \& Remillard, R. A. 2006, ApJ, 636, L113

Sobczak, G. J., McClintock, J. E., Remillard, R. A., Cui, W., Levine, A. M., Morgan, E. H., Orosz, J. A., \& Bailyn, C. D. 2000a, ApJ, 531, 537

Strohmayer, T., \& Bildsten, L. 2006, in "Compact Stellar X-ray Sources", eds. W. H. G. Lewin \& M. van der Klis, (Cambridge: Cambridge U. Press), 113

Strohmayer, T., Markwardt, C. B., Swank, J. H., \& in't Zand, J. 2003, ApJ, 596, L67

Taam, R. E., Woosley, S. E., \& Lamb 1996, ApJ 459, 271

Tomsick, J. A., Kalemci, E., Corbel, S., \& Kaaret, P. 2003, ApJ, 592, 1100

Tournear, D., et al. 2003, ApJ, 595, 1058; T03

Thorsett, S. E., \& Chakrabarty, D. 1999, ApJ, 512,288

van der Klis, M. 2006, in "Compact Stellar X-ray Sources," eds. W. H. G. Lewin \& M. van der Klis, (Cambridge: Cambridge U. Press), 39

Werner, N. et al. 2004, A\&A, 416, 311

Wijnands, R., \& van der Klis, M. 1999, ApJ, 522, 965

Wijnands, R., Muno, M. P., Miller, J. M., Franco, L. M., Strohmayer, T., Galloway, D., \& Chakrabarty, D. 2002, ApJ, 566, 1060

Wijnands, R., et al. 2005, ApJ, 618, 883

Woosley, S. E., et al. 2004, ApJS, 151, 75

Woosley, S. E., \& Taam, R. E. 1976, Nature, 263, 101

Yuan, Y-F., Narayan, R., \& Rees, M. J. 2004, ApJ, 606, 1112

This 2-column preprint was prepared with the AAS LATEX macros v5.2. 
TABLE 1

Non-PUlsing Galactic X-Ray Transients: 1996-2004

\begin{tabular}{|c|c|c|c|c|c|c|c|c|c|}
\hline $\begin{array}{l}\text { X-ray } \\
\text { name }\end{array}$ & $\begin{array}{l}\text { No. } \\
\text { obs. }\end{array}$ & $\begin{array}{l}\operatorname{archive} \\
(\mathrm{ks})^{\mathrm{a}}\end{array}$ & $\begin{array}{c}\text { anal. } \\
(\mathrm{ks})^{\mathrm{b}}\end{array}$ & $\begin{array}{c}d \\
(\mathrm{kpc})\end{array}$ & $\begin{array}{l}\operatorname{mass} \\
\left(M_{\odot}\right)\end{array}$ & $\begin{array}{c}N_{H} \\
\left(10^{22}\right)\end{array}$ & $\begin{array}{c}i \\
\text { (deg.) }\end{array}$ & $\begin{array}{l}\text { cand. } \\
\text { bursts }^{\mathrm{c}}\end{array}$ & $\begin{array}{l}\text { type I } \\
\text { bursts }\end{array}$ \\
\hline \multicolumn{10}{|c|}{ Black Holes (BHBs) } \\
\hline \multicolumn{10}{|l|}{ Black Holes (BHBs) } \\
\hline XTE J1118+480 & 53 & 152 & 143 & 1.8 & 6.8 & 0.012 & 70 & 0 & 0 \\
\hline $4 \mathrm{U} 1354-64$ & 10 & 67 & 65 & 27.0 & 10.0 & 1.1 & 60 & 0 & 0 \\
\hline $4 \mathrm{U} 1543-47$ & 109 & 271 & 260 & 7.5 & 9.4 & 0.4 & 21 & 0 & 0 \\
\hline XTE J1550-564 & 421 & 1233 & 1030 & 5.3 & 9.6 & 0.9 & 72 & 3 & 0 \\
\hline XTE J1650-500 & 183 & 358 & 345 & 7.0 & 5.5 & 0.67 & 60 & 2 & 0 \\
\hline GRO J1655-40 & 81 & 388 & 377 & 3.2 & 6.3 & 0.9 & 70 & 0 & 0 \\
\hline GX339-4 & 586 & 1749 & 1061 & 4.0 & 7.0 & 0.6 & 50 & 0 & 0 \\
\hline V4641 Sgr & 68 & 172 & 154 & 9.8 & 7.1 & var. & 66 & 2 & 0 \\
\hline XTE J1859+226 & 133 & 386 & 365 & 11.0 & 9.8 & 0.4 & 60 & 0 & 0 \\
\hline \multicolumn{10}{|c|}{ BH Candidates (BHCs) } \\
\hline \multicolumn{10}{|c|}{ BH Candidates (BHCs) } \\
\hline $4 \mathrm{U} 1630-47$ & 595 & 1294 & 1156 & 8.5 & 8.0 & 8.8 & 70 & 8 & 0 \\
\hline H $1743-322$ & 256 & 906 & 822 & 8.5 & 8.0 & 2.2 & 60 & 5 & 0 \\
\hline SAX J1711.6-3808 & 18 & 47 & 45 & 8.5 & 8.0 & 2.8 & 60 & 0 & 0 \\
\hline XTE J1720-318 & 102 & 301 & 288 & 8.5 & 8.0 & 1.2 & 60 & 0 & 0 \\
\hline GRS 1739-278 & 11 & 30 & 27 & 8.5 & 8.0 & 1.7 & 60 & 0 & 0 \\
\hline SLX 1746-331 & 51 & 149 & 145 & 8.5 & 8.0 & 1.8 & 60 & 0 & 0 \\
\hline XTE J1748-288 & 24 & 106 & 103 & 8.5 & 8.0 & 8.0 & 60 & 0 & 0 \\
\hline XTE J1755-324 & 4 & 12 & 10 & 8.5 & 8.0 & 0.6 & 60 & 0 & 0 \\
\hline XTE J1908+094 ${ }^{\mathrm{d}}$ & 32 & 41 & 0.0 & $\ldots$ & $\ldots$ & $\ldots$ & $\ldots$ & $\ldots$ & $\ldots$ \\
\hline XTE J2012+381 & 24 & 60 & 58 & 8.5 & 8.0 & 0.7 & 60 & 0 & 0 \\
\hline \multicolumn{10}{|c|}{ Neutron Star Bursters } \\
\hline Neutron Star Burst & & & & & & & & & \\
\hline 4U 1608-52 & 378 & 1451 & 1387 & 3.6 & 1.4 & 1.0 & 60 & 26 & 26 \\
\hline X1658-298 & 79 & 355 & 351 & 10.0 & 1.4 & 2.0 & 77 & $26^{\mathrm{e}}$ & 26 \\
\hline RX J1709.5-2639 & 38 & 142 & 139 & 8.5 & 1.4 & 0.3 & 60 & 3 & 3 \\
\hline X1711-339 & 10 & 42 & 39 & 7.5 & 1.4 & 1.5 & 60 & 0 & 0 \\
\hline XTE J1723-376 & 2 & 20 & 19 & 8.5 & 1.4 & 4.0 & 72 & 2 & 2 \\
\hline SAX J1747.0-2853 & 9 & 42 & 41 & 7.5 & 1.4 & 8.8 & 60 & 0 & 0 \\
\hline EXO $1745-248$ & 52 & 149 & 145 & 8.7 & 1.4 & 3.0 & 60 & 24 & 22 \\
\hline $\mathrm{X} 1744-361$ & 10 & 39 & 38 & 8.5 & 1.4 & 2.5 & 60 & 0 & 0 \\
\hline
\end{tabular}



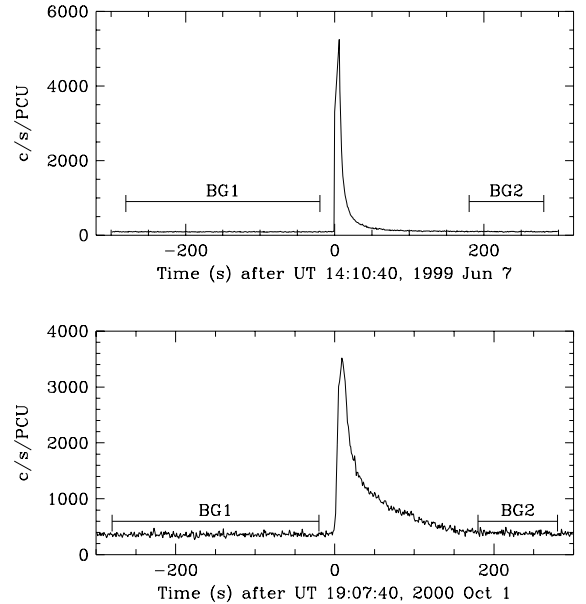

Fig. 1. - Sample of a short (top panel) and long (bottom) X-ray burst from Aql X-1. The time references are the burst trigger time, or the first second where Eq. 1 is true for 7 consecutive s. These events would trigger bursts for times $>20$ consecutive s.

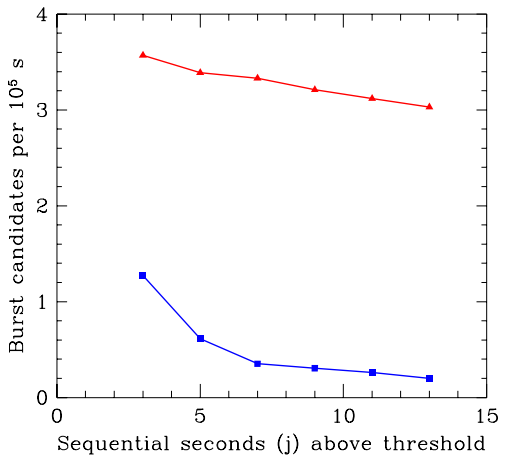

Fig. 2.- Rate of finding burst candidates as a function of the number of sequential seconds for which the variance test for excess flux (Eq. 1) is satisfied. Triangles denote the NS group, while squares represent the combined results for BHBs and BHCs.

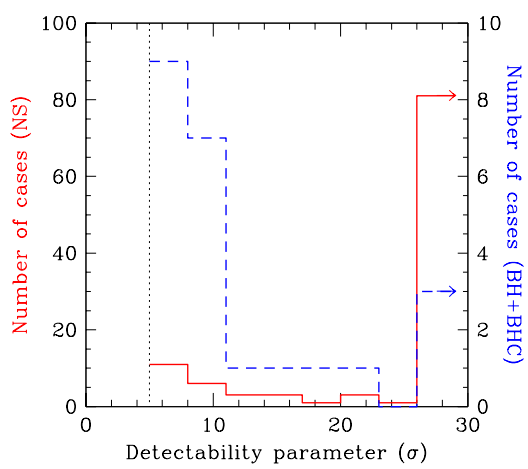

Fig. 3.- The detectability of burst candidates is quantified by sweeping the search window over the burst to find the maximum signal-to-noise ratio of the $j$ th brightest point above the background (see text). These histograms are made for the burst candidates that were identified using $j=7$ s. Most of the burst candidates for the group of combined BHBs and BHCs (blue, dashed line) have detectability that is close to the $5 \sigma$ threshold, which is shown as a dotted vertical line. In contrast, the burst candidates for neutron stars (red, solid line) have much higher significance. The horizontal axis is truncated to allow comparison of the two histograms, and the arrows for the last data points indicate that the plotted number includes all cases from the lower edge of the bin $(26 \sigma)$ to the maximum observed value. 
TABLE 1-Continued

\begin{tabular}{|c|c|c|c|c|c|c|c|c|c|}
\hline $\begin{array}{l}\text { X-ray } \\
\text { name }\end{array}$ & $\begin{array}{l}\text { No. } \\
\text { obs. }\end{array}$ & $\begin{array}{c}\text { archive } \\
(\mathrm{ks})^{\mathrm{a}}\end{array}$ & $\begin{array}{c}\text { anal. } \\
(\mathrm{ks})^{\mathrm{b}}\end{array}$ & $\begin{array}{c}d \\
(\mathrm{kpc})\end{array}$ & $\begin{array}{l}\text { mass } \\
\left(M_{\odot}\right)\end{array}$ & $\begin{array}{c}N_{H} \\
\left(10^{22}\right)\end{array}$ & $\begin{array}{c}i \\
\text { (deg.) }\end{array}$ & $\begin{array}{l}\text { cand. } \\
\text { bursts }^{\mathrm{c}}\end{array}$ & $\begin{array}{l}\text { type I } \\
\text { bursts }\end{array}$ \\
\hline $\mathrm{X} 1745-203$ & 18 & 130 & 128 & 8.5 & 1.4 & 0.6 & 60 & 16 & 16 \\
\hline SAX J1750.8-2900 & 12 & 48 & 47 & 5.2 & 1.4 & 2.0 & 60 & 4 & 4 \\
\hline X1803-245 & 11 & 35 & 33 & 7.6 & 1.4 & 2.0 & 60 & 0 & 0 \\
\hline SAX J1810.8-2609 & 0 & 0 & 0 & $\cdots$ & $\cdots$ & $\cdots$ & $\cdots$ & $\cdots$ & $\cdots$ \\
\hline AQL X-1 & 297 & 1297 & 1258 & 5.0 & 1.4 & 0.3 & 60 & 31 & 31 \\
\hline XTE J2123-058 & 5 & 67 & 66 & 9.6 & 1.4 & 0.06 & 60 & 5 & 5 \\
\hline \multicolumn{10}{|c|}{ Unclassified } \\
\hline \multicolumn{10}{|l|}{ Unclassified } \\
\hline SAX J1428.6-5422 & 18 & 37 & 0 & $\cdots$ & $\cdots$ & $\cdots$ & $\cdots$ & $\cdots$ & $\cdots$ \\
\hline XTE J1739-285 & 7 & 21 & 0 & $\cdots$ & $\cdots$ & $\cdots$ & $\cdots$ & $\cdots$ & $\cdots$ \\
\hline SAX J1805.5-2031 & 45 & 70 & 0 & $\ldots$ & $\ldots$ & $\ldots$ & $\ldots$ & $\ldots$ & $\ldots$ \\
\hline XTE J1856+053 & 2 & 8 & 0 & $\cdots$ & $\cdots$ & $\cdots$ & $\cdots$ & $\cdots$ & $\cdots$ \\
\hline
\end{tabular}

${ }^{\text {a }}$ RXTE total archive, as of 2004 November 1.

${ }^{\mathrm{b}}$ We analyzed the portion of the archive that is publicly available, shows a source flux above $5.0 \mathrm{c} \mathrm{s}^{-1}$ $\mathrm{PCU}^{-1}$, and is free of systematic problems.

${ }^{c}$ These triggers are based on timing analyses, with a requirement for 7 sequential seconds that a burst candidate is above the $5 \sigma$ trigger threshold (see text).

${ }^{\mathrm{d}}$ Source excluded since there is another known X-ray source in the PCA field of view.

${ }^{\mathrm{e}}$ One burst was added because X-ray eclipses and absorption dips interfere with the variance test for detecting burst candidates.

TABLE 2

Probabilities OF OBSERVING NO BURSTS From the GROUP OF BHBS

\begin{tabular}{c|ccc}
\hline \hline$T_{\text {IBC }}$ & $R / R_{\mathrm{S}}=9 / 8$ & $R / R_{\mathrm{S}}=2$ & $R / R_{\mathrm{S}}=3$ \\
\hline $10^{5.0} \mathrm{~K}$ & $3 \times 10^{-16}$ & $1 \times 10^{-16}$ & $1 \times 10^{-7}$ \\
$10^{5.5} \mathrm{~K}$ & $3 \times 10^{-16}$ & $1 \times 10^{-16}$ & $1 \times 10^{-7}$ \\
$10^{6.0} \mathrm{~K}$ & $3 \times 10^{-16}$ & $1 \times 10^{-16}$ & $1 \times 10^{-7}$ \\
$10^{6.5} \mathrm{~K}$ & $3 \times 10^{-16}$ & $6 \times 10^{-17}$ & $9 \times 10^{-8}$ \\
$10^{7.0} \mathrm{~K}$ & $3 \times 10^{-16}$ & $3 \times 10^{-17}$ & $1 \times 10^{-7}$ \\
$10^{7.5} \mathrm{~K}$ & $4 \times 10^{-16}$ & $5 \times 10^{-17}$ & $1 \times 10^{-7}$ \\
$10^{8.0} \mathrm{~K}$ & $4 \times 10^{-16}$ & $4 \times 10^{-17}$ & $1 \times 10^{-7}$ \\
\hline
\end{tabular}


TABLE 3

Probabilities of OBSERVING NO BURSTS FROM THE COMBINED GROUP OF BHBs AND BHCS

\begin{tabular}{c|ccc}
\hline \hline$T_{\text {IBC }}$ & $R / R_{\mathrm{S}}=9 / 8$ & $R / R_{\mathrm{S}}=2$ & $R / R_{\mathrm{S}}=3$ \\
\hline $10^{5.0} \mathrm{~K}$ & $3 \times 10^{-39}$ & $5 \times 10^{-35}$ & $2 \times 10^{-13}$ \\
$10^{5.5} \mathrm{~K}$ & $3 \times 10^{-39}$ & $5 \times 10^{-35}$ & $2 \times 10^{-13}$ \\
$10^{6.0} \mathrm{~K}$ & $3 \times 10^{-39}$ & $5 \times 10^{-35}$ & $2 \times 10^{-13}$ \\
$10^{6.5} \mathrm{~K}$ & $3 \times 10^{-39}$ & $2 \times 10^{-35}$ & $1 \times 10^{-13}$ \\
$10^{7.0} \mathrm{~K}$ & $3 \times 10^{-39}$ & $5 \times 10^{-36}$ & $2 \times 10^{-13}$ \\
$10^{7.5} \mathrm{~K}$ & $4 \times 10^{-39}$ & $1 \times 10^{-35}$ & $2 \times 10^{-13}$ \\
$10^{8.0} \mathrm{~K}$ & $4 \times 10^{-39}$ & $1 \times 10^{-35}$ & $2 \times 10^{-13}$ \\
\hline
\end{tabular}
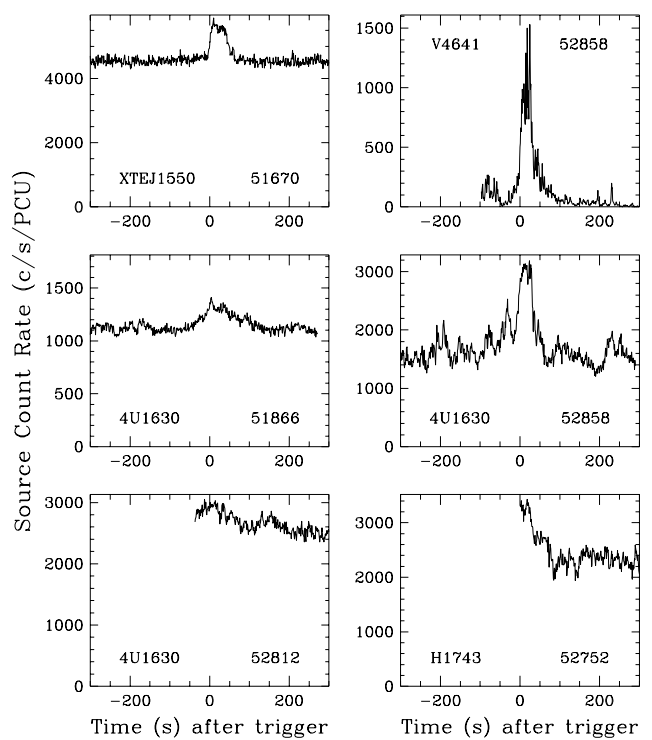

Fig. 4.- Samples of the burst candidates with durations $>30 \mathrm{~s}$ for the BHB and BHC groups. Each panel is labeled by the source name (abbreviated) and the observation day (MJD). The time axis is referenced to the burst trigger time. None of these events was confirmed as a type I X-ray burst. In each case the spectrum of the excess flux is inconsistent with a blackbody spectrum.
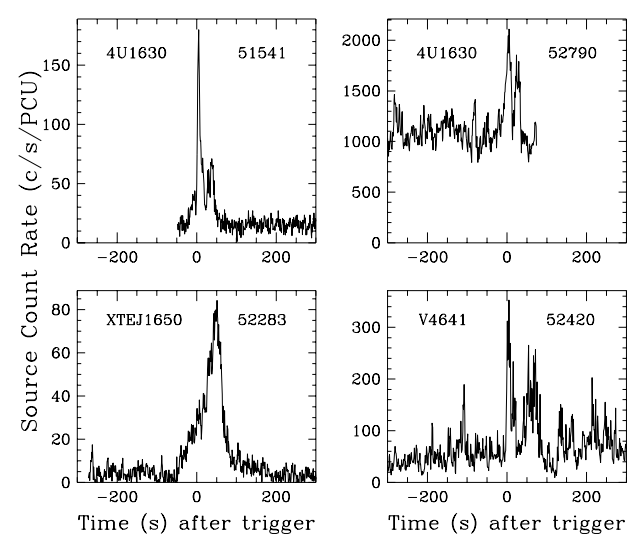

Fig. 5. - Samples of the BH burst candidates with short duration. The plot labels follow the convention of Fig. 4. None of these events is a type I X-ray burst that would signify a thermonuclear explosion on the surface of a compact object. 


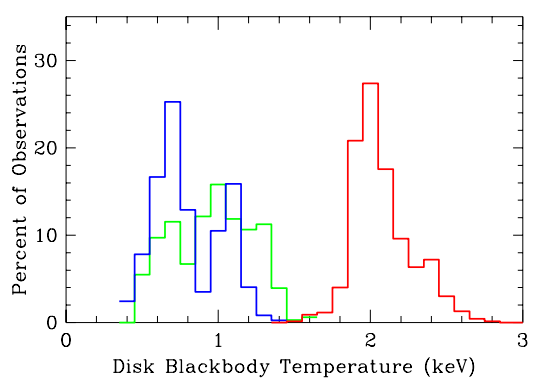

Fig. 6.- Normalized distributions in the temperatures of the thermal component for different groups of compact objects. Higher temperatures are seen for the NS group (red line), selecting the soft-state observations of 11 sources (excluding the two dippers). The groups of BHBs (blue line) and BHCs (green line) show systematically lower temperatures. These data are also selected for soft $\mathrm{X}$-ray color, and they additionally satisfy conditions of the thermal state given by McClintock \& Remillard (2006). This excludes soft-state observations that are in the "steep power law" state, which is a spectral state not observed in NS systems.

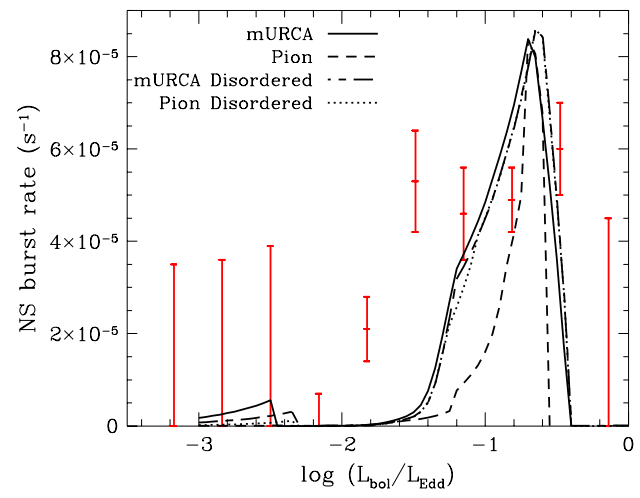

Fig. 7.- Average burst rates (red points; $1 \sigma$ error bars) for the NS group, binned as a function of the bolometric luminosity divided by the Eddington luminosity. The burst models all assume a mass of $1.4 M_{\odot}$, radius of $10.4 \mathrm{~km},\langle\dot{M}\rangle$ equal to $10^{-3} \dot{M}_{\mathrm{Edd}}$, and the distances given in Table 1 . The burst rates predicted by four burst models are shown. The Pion model is ruled out, but the other three models are generally consistent with the data. 


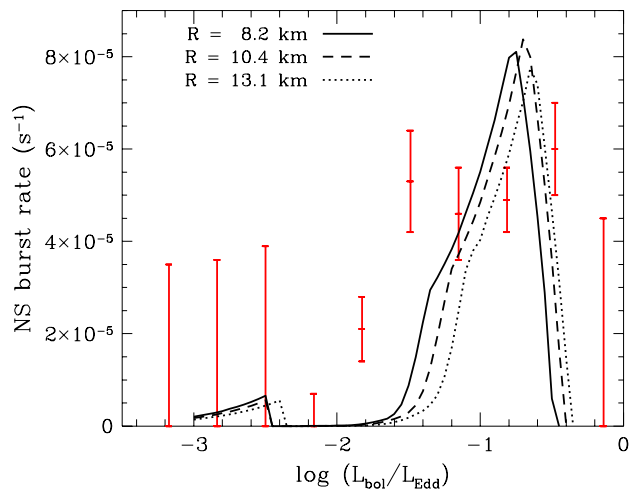

Fig. 8.- Average burst rates for the NS group, as defined for Fig. 6. Here, the models vary the NS radius, while assuming a mass of $1.4 M_{\odot},\langle\dot{M}\rangle$ equal to $10^{-3} \dot{M}_{\text {Edd }}$, mUrca cooling in the core, an ordered crust, and the distances given in Table 1 . The models are all roughly consistent with the data.

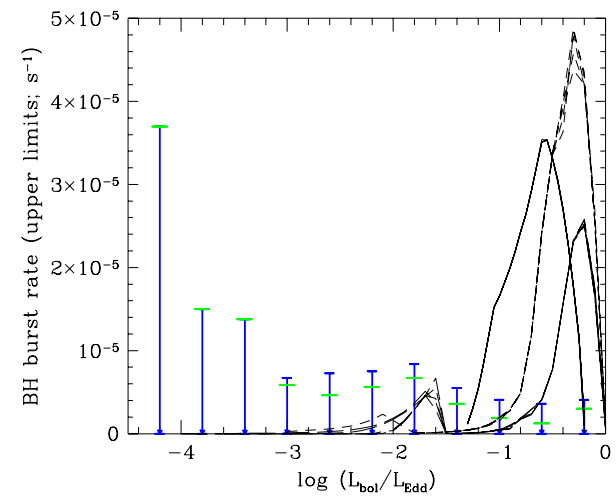

Fig. 9. - Upper limits (90\% confidence) for burst rates in BHBs (upper/blue error bars), and for the combined BHB and BHC groups (lower/green error bars), binned as a function of the bolometric luminosity divided by the Eddington luminosity. The burst rates predicted for a heavy compact object of mass $8 M_{\odot}$ with a solid surface are shown by the curves. The three groups of curves correspond to different assumptions for the radius: $R / R_{\mathrm{S}}=3$ (the shortest peak on the right), 2 (the tallest middle peak), and $9 / 8$ (the intermediate height peak on the left). In each group seven models were considered: $T_{\mathrm{IBC}}=10^{5}$ to $10^{8} \mathrm{~K}$, with steps of 0.5 in logarithmic units. The narrowness of each group of seven overplotted curves at large values of $L_{\mathrm{bol}} / L_{\mathrm{Edd}}$ illustrates the insensitivity of the burst rates to the inner temperature boundary condition. 